ít xâm lấn dài hơn có ý nghĩa thống kê so với phẫu thuật kinh điển, đặc biệt đối với sửa van hai lá. Vì vậy, trong giai đoạn đâu chúng tôi chọn những bệnh nhân có tổn thương van hai lá đơn giản, thường là sa lá sau phần $\mathrm{P} 2$, chức năng thất trái tốt, không hoặc tăng nhẹ áp lực động mạch phổi. Về sau khi đã vượt qua đường cong huấn luyện, chúng tôi có thể mở rộng chỉ định cũng như phương pháp mổ. Tuy nhiên, với những trường hợp tổn thương van phức tạp mà vẫn còn khả năng sửa, chúng tôi ưu tiên dùng phương pháp mổ mở để sửa van.

Phẫu thuật sửa van hai lá 1 trong những phẫu thuật đòi hỏi nhiều kĩ năng nhất trong các phẫu thuật tim có thể thực hiên được bằng phương pháp ít xâm lấn. So với thay van hai lá, phẫu thuật sửa van hai lá đem lại nhiều lợi ích vượt trội cho người bệnh: Cải thiện chức năng thất trái, giảm tî lệ tử vong sớm và dài hạn, giảm các biến chứng do việc sử dụng thuốc kháng đông loại kháng Vitamin $\mathrm{K}$ lâu dài, giảm tỉ lệ phẫu thuật lại. Chúng tôi tin rằng cùng với sự phát triển của khoa học kĩ thuật, đặc biệt ứng dụng của các hệ thống robot đã triển khai ở các nước phát triển, chất lượng của phẫu thuật nội soi sửa van hai lá sẽ ngày càng được cải thiện và nâng cao.

\section{KẾT LUẬN}

Phẫu thuật sửa van hai lá ít xâm lấn bằng đường mở ngực nhỏ có nội soi lồng ngực hỗ trợ khả thi, an toàn và có kết quả sớm cũng như trung hạn khả quan tại bệnh viện của chúng tôi. Tuy nhiên vẫn còn những thách thức kĩ thuật và đòi hỏi đường cong huấn luyện dài cho nên cần có sự đầu tư nhân lực cũng như trang thiết bị. Việc chọn lựa bệnh nhân hợp lý và tuân thủ các quy định về an toàn phẫu thuật sẽ giúp đem lại kết quả tốt và đảm bảo sự an toàn cho người bệnh.

\section{TÀI LIỆ THAM KHẢO}

1. Carpentier A.F., Adams D.H., Filsoufi F. và cộng sứ. (2010), Carpentier's reconstructive valve surgery: from valve analysis to valve reconstruction, Saunders Elsevier, Maryland Heights, Mo.

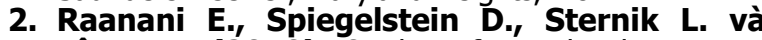
cộng sự. (2010). Quality of mitral valve repair: Median sternotomy versus port-access approach. The Journal of Thoracic and Cardiovascular Surgery, 140(1), 86-90.

3. Cao C., Gupta S., Chandrakumar D. và cộng sứ. (2013). A meta-analysis of minimally invasive versus conventional mitral valve repair for patients with degenerative mitral disease. Annals of cardiothoracic surgery, 2(6), 11.

4. McClure R.S., Cohn L.H., Wiegerinck E. và cộng sự. (2009). Early and late outcomes in minimally invasive mitral valve repair: An elevenyear experience in 707 patients. The Journal of Thoracic and Cardiovascular Surgery, 137(1), 70-75.

5. Davierwala P.M., Seeburger J., Pfannmueller B. và cộng sự. (2013). Minimally invasive mitral valve surgery: "The Leipzig experience". Annals of cardiothoracic surgery, 2(6), 7.

6. Sündermann S.H., Sromicki J., Rodriguez Cetina Biefer H. và công sứ. (2014). Mitral valve surgery: Right lateral minithoracotomy or sternotomy? A systematic review and metaanalysis. The Journal of Thoracic and Cardiovascular Surgery, 148(5), 1989-1995.e4.

7. Falk V., Cheng D.C.H., và Martin J. (2011) Minimally Invasive versus Open Mitral Valve Surgery a Consensus Statement of the International Society of Minimally Invasive Coronary Surgery (ISMICS) 2010. 6(2), 11.

\title{
VAI TRÒ CỦA KHOẢNG THỜI GIAN TPEAK - TEND TRONG PHÂN TẦ NGUY CƠ Ở BÊ̂NH NHÂN CÓ ĐIỆN TÂM ĐỒ DANG BRUGADA
}

\section{TÓM TẮT}

Mục tiêu: (1) Khảo sát khoảng thời gian TpeakTend và tỉ lệ Tpeak-Tend/QT ở bệnh nhân có điện tâm

\footnotetext{
${ }^{1}$ Trường Đai Hoc Y Hà Nôi

2 Viện Tim mạch Việt Nam - Bệnh viện Bạch Mai

${ }^{3}$ Trường Đại Học Y Dược-Đại học Quốc Gia Hà Nội.

Chịu trách nhiếm chính: Phạm Trần Linh

Email: ptlinhmd@gmail.com

Ngày nhận bài: 29.7.2021

Ngày phản biên khoa họ: 30.9.2021

Ngày duyệt bài: 5.10 .2021
}

\section{Phạm Trung Hiếu 1,2, Phạm Trần Linh ${ }^{2,3}$, Viên Hoàng Long,3, Lê Thị Lan Hương1.}

đồ dạng Brugada. (2)Đánh giá giá trị của khoảng thời gian Tpeak- Tend và tî lê Tpeak- Tend / QT trong phân tâng nguy cơ rối loạn nhịp thất ở những bệnh nhân trên. Phương pháp nghiên cứu: Nghiên cứu mô tả cắt ngang 41 bệnh nhân có điện tâm đồ dạng Brugada được tiến hành thăm dò điện sinh lý tại Viên Tim Mạch - Bệnh viện Bạch Mai. So sánh khoảng thời gian TpTe và tỉ lệ TpTe/QT với kết quả thăm dò điện sinh lý, tìm mối liên quan. Kết quả: 41 bệnh nhân có điên tâm đồ dang Brugada tham gia nghiên cứu có độ tuổi trung bình là $49 \pm 11$ tuổi, nam giới chiếm 95,1\%. Có 24 bệnh nhân (chiếm $58.55 \%$ ) thăm dò điên sinh lý dương tính gây nhịp nhanh thất đa hình thái, rung thất. Khoảng thời gian Tpeak-Tend dài hơn đáng kể ở 
nhóm bệnh nhân dương tính so với nhóm bệnh nhân âm tính trong thăm dò điện sinh lý tim. Kết luận: Khoảng thời gian Tpeak-Tend và tỉ lệ Tpeak-Tend/QT ở chuyển đạo V1 và V2 tăng cao hơn đáng kể ở những bệnh nhân Brugada gây được cơn rối loạn nhịp thất nguy hiểm trong thăm dò điện sinh lý tim.

Tư khoá: Hội chứng Brugada, khoảng thời gian Tpeak - Tend, phân tầng nguy cơ.

\section{SUMMARY}

\section{ROLE OF TPEAK-TEND INTERVAL IN RISK STRATIFICATION FOR PATIENTS WITH BRUGADA ECG PATTERN}

Background: Brugada syndrome is one of the serious arrhythmias, which may cause syncope or sudden death. The standard 12-lead electrocardiogram (ECG) is usually used to detect Brugada syndrome. Nowaday, the utility of electrophysiology for the risk stratification of sudden death for patients with Brugada ECG pattern has been controversial regarding sensitivity and specificity. Tpeak-Tend interval and Tpeak-Tend/QT ratio, which are measured by using the surface ECG, can predict the risk of arrhythmias. Objectives: (1) Investigate the Tpeak-Tend interval and the Tpeak-Tend/QT ratio in patients with Brugada ECG pattern. (2) Evaluate Tpeak-Tend interval and the Tpeak-Tend/QT ratio as the risk stratification of ventricular arrhythmias in these patients. Subjects and methods: A descriptive cross-sectional study on 41 patients with Brugada ECG pattern from Vietnam National Heart Institute - Bach Mai Hospital. The patients were indicated for electrocardiogram, echocardiogram, basic blood test, and taken personal and family history. Seeking the relationship by comparing the measurement results on the standard 12-lead electrocardiogram with the results of EP. Results: 41 patients (95,1\% male) with Brugada ECG pattern participating in the study had an average age of $49 \pm 11$ years old. EPS was positive in $58.55 \%$ (24 patients), which induced the ventricular tachycardia, ventricular fibrillation. The Tpeak-Tend interval was significantly longer in the positive EPS group than in the EP negative group. Conclusions: Tpeak-Tend interval and Tpeak-Tend/QT ratio were significantly higher in leads $\mathrm{V} 1$ and $\mathrm{V} 2$ in Brugada patients, who had an life-threatening ventricular arrhythmia during EPS.

Keywords: Brugada syndrome, Tpeak - Tend interval, risk stratification.

\section{I. ĐẠTT VẤN ĐỀ}

Vào những năm đầu thập niên 90 của thế kỉ trước, anh em nhà Brugada là Joshep Brugada và Pedro Brugada đã nhận thấy 4 trường hợp đột tử với biểu hiện điện tầm đồ giống nhau.Đển nằm 1992, với bài báo mô tả về 8 trường hợp tương tự, được công bố trên tạp chí "The American College of Cardiology", đã mở ra cánh cửa nghiên cứu về một căn bệnh hoàn toàn mới, có tên là "Hội chứng Brugada"[1].

Trong số những bệnh nhân có điện tâm đồ dạng Brugada, phần lớn ở thể không có triệu chứng. Không may là đối với hầu hết bệnh nhân, triêu chứng đâuu tiên là ngừng tim hoặc đột tử do tim[2]. Do đó việc phân tâng nguy cớ với những bệnh nhân có điện tâm đồ dạng Brugada vô cùng quan trọng. Thăm dò điện sinh lý vẫn là một phương pháp phân tầng nguy cơ được thực hiển rộng rãi trên các trung tâm tim mạch trển thế giới, tuy nhiên đây một thủ thuật thăm dò có xâm lấn và còn những tranh luận về độ nhậy và độ đặc hiệu đối với việc chẩn đoán và phân tầng nguy cơ[3]. Trong khi đó, điện tâm đồ 12 chuyển đạo vẫn là công cụ mang tính chất quyết định và không thể thay thế được trong chẩn đoán. Hiện nay nghiên cứu về $\mathrm{BrS}$ trong nước còn khá khiêm tốn, chưa có nghiên cứu phân tầng nguy cơ rối loạn nhịp bằng phương pháp thăm dò không xâm lấn. Vì vậy chúng tôi thực hiện đề tài: "Vai trò của khoảng thời gian Tpeak - Tend trong phân tầng nguy cơ ở bệnh nhân có điện tâm đồ dạng Brugada".

\section{II. ĐỐl TƯỢNG VÀ PHƯƠNG PHÁP NGHIÊN CỨU}

Đối tượng nghiên cứu. Các bệnh nhân có điện tâm đồ dạng Brugada thoả mãn tiêu chuẩn lựa chọn vào mẫu được thăm dò điện sinh lý tim để đánh giá nguy cơ xuất hiện các rối loạn nhịp thất nguy hiểm tại Viện Tim mạch - Bệnh viện Bạch Mai từ 7/2020 đến 7/2021.

Phương pháp nghiên cứu. Nghiên cứu mô tả cắt ngang với cỡ mẫu thuận tiện.

Xử lý số liệu nghiên cứu. Các số liệu thu thập được của nghiên cứu được xử lý theo các thuật toán thống kê y học trên máy vi tính bằng chương trình phần mềm SPSS 20.0 để tính toán các thông số: trung bình, phương sai, độ lệch chuẩn. Giá trị $p<0,05$ được coi là có ý nghĩa thống kê.

\section{KẾT QUẢ NGHIÊN CỨU}

\section{1 Đặc điểm chung của nhóm bệnh} nhân nghiên cứu. Trong nghiên cứu có 41 bệnh nhân điện tâm đồ dạng Brugada với độ tuổi trung bình là $49 \pm 11$ tuổi. 39 bệnh nhân là nam giới, chiếm $95,1 \%$ và chỉ có 2 bệnh nhân là nữ giới, chiếm 4,9\%.Có 4 bệnh nhân trong nghiên cứu có người thân trong gia đình thuộc thế hệ thứ 1 đột tử trẻ tuổi (< 45 tuổi), chiếm 9,75\%.

- Bệnh nhân có điện tâm đồ Brugada type 1 trong nghiên cứu có tỉ lệ nhiều nhất, là 24 bệnh nhân, chiếm 58,5\%.Bệnh nhân có điện tâm đồ Brugada type 2 và type 3 trong nghiên cứu chiếm tỉ lệ lần lượt là $26,8 \%$ và $14,7 \%$.

- Có 24 bệnh nhân (chiếm 58,55\%) thăm dò điện sinh lý dương tính gây nhịpnhanh thất / 
rung thất, không có sự khác biệt đáng kể khi kích thích tim tại 2 vị trí mỏm thất phải và đường ra thất phải.

3.2. Khoảng thời gian Tpeal-Tend và trị số tỉ lệ TpeakTend/QT

- Khoảng thời gian trung bình Tpeak-Tend ở chuyển đạo V1 là $77,12 \pm 6,645 \mathrm{~ms}$, V2 là 78,66 $\pm 7,6 \mathrm{~ms}$,V3 là $77,95 \pm 6 \mathrm{~ms}$, V4 là $75,95 \pm$ $6,74 \mathrm{~ms}$, V5 là 76,39 $\pm 7,2 \mathrm{~ms}$ và tỉ lệ trung bình TpeakTend/QT ở V1 là $0,203 \pm 0,016$, V2 là $0,204 \pm 0,015$, V3 là $0,21 \pm 0,016$, V4 là $0,215 \pm$ 0,017, V5 là $0,216 \pm 0,019$.

- Trị số tî lệ trung bình TpTe/QT ở chuyển đạo V1 là $0,203 \pm 0,016$, chuyển đạo V2 là $0,204 \pm 0,015$, chuyển đạo V3 là $0,21 \pm 0,016$, chuyển đạo V4 là $0,215 \pm 0,017$, chuyển đạo V5 là $0,216 \pm 0,019$.

3.3. Mối tương quan giữa khoảng thời gian Tpeak-Tend và tỉ lệ TpeakTend/QT với thăm dò điện sinh lý tim

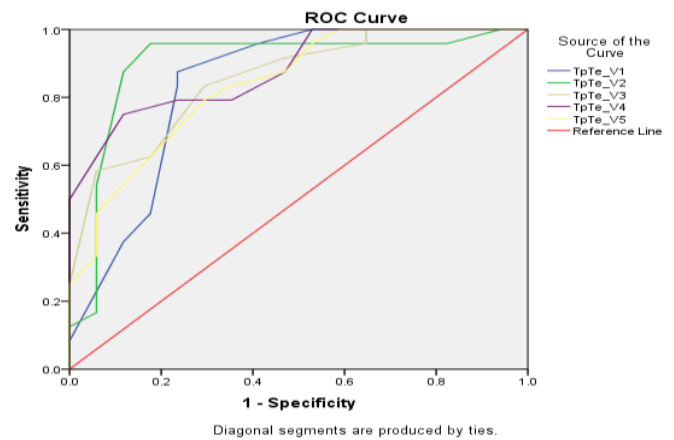

Biểu đồ 1. Diên tích dưới đường cong ROC giữa trị số thời gian Tpeak - Tend với thăm dò điên sinh lý tim

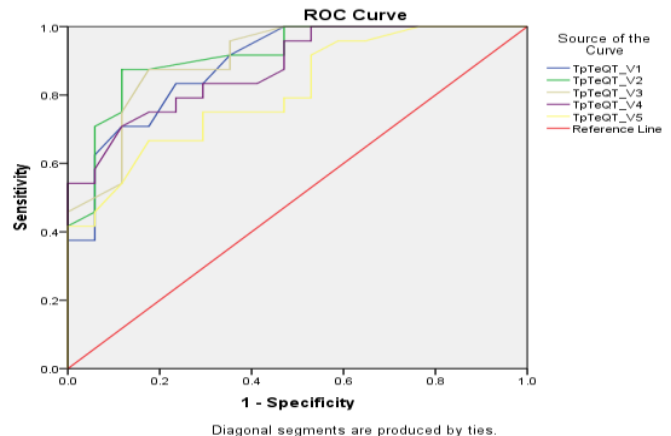

Biểu đồ 2. Diện tích dưới đường cong ROC giứa trị số tí lệ TpTe /QT với thăm dò điện sinh lý

Khoảng thời gian Tpeak-Tend và trị số tỉ lệ TpeakTend/ QT tại các chuyển đạo từ V1 đến V5 đều có giá trị tiên lượng được kểt quả gây được cơn nhịp nhanh thất/ rung thất khi kích thích tim theo chương trình trong thăm dò điện sinh lý.

\section{BÀN LUÂN}

Nghiên cứu của chúng tôi có 41 bệnh nhân điện tâm đồ dạng Brugada với độ tuổi trung bình là $49 \pm 11$ năm,bệnh nhân trẻ tuổi nhất là 27 tuổi và bệnh nhân lớn tuổi nhất là 68 tuổi.Báo cáo của hội nghị đồng thuận lần thứ 2 được xác nhận bởi HRS/EHRA về độ tuổi trung bình của bệnh nhân $\mathrm{BrS}$ hay bị đột tử là $41 \pm 15$ năm. Bệnh nhân trẻ tuổi nhất được chẩn đoán là 2 ngày tuổi và bệnh nhân lớn tuổi nhất là 84 tuổi[4]. Bệnh nhân nam giới chiếm chủ yếu với $95,1 \%$ so với bệnh nhân nữ giới là $4,9 \%$ trong nghiên cứu. Bệnh nhân có điện tâm đồ Brugada type 1 chiếm tỉ lệ nhiều nhất là $58,5 \%$. Các nghiên cứu đã thấy rằng Tpeak trùng với kết thúc tái phân cực sớm nhất, trong khi Tend trùng với kết thúc muộn nhất của tái phân cực. Điều này cho thấy phân tán toàn bộ tái cực được thể hiện bởi khoảng thời gian Tpeak-Tend[5]. Chúng tôi đã thực hiện phương pháp đo tiếp tuyến để tính khoảng thời gian Tpeak-Tend, kết quả là trung bình cộng của 3 nhịp liên tiếp từ chuyển đạo V1 đến V5. Tỉ lệ TpTe /QT được xem là một chỉ số nhạy cảm hơn của rối loạn nhịp tim so với khoảng thời gian Tpeak - Tend vì nó cung cấp ước tính phân tán tái cực liên quan đến tổng thời gian tái cực[6].

Chúng tôi sữ dụng phương pháp đường cong ROC để tìm mối tương quan giữa khoảng thời gian Tpeak-Tend và trị số tỉ lệ Tpeak-Tend/QT với kết quả thăm dò điện sinh lý thấy rằng khoảng thời gian TpTe và tỉ lệ TpTe/QT tại các chuyển đạo từ V1 đến V5 đều có giá trị tiên lượng được kết quả gây được cơn VF/VT trong thăm dò điện sinh lý tim. Khoảng thời gian TpTe dài hơn đáng kể ở nhóm bệnh nhân dương tính so với nhóm bệnh nhân âm tính trong $E P$, như tại chuyển đạo V1 $(79,83 \pm 6,78 \mathrm{~ms}$ với 73,29 \pm $4,21 \mathrm{~ms} ; \mathrm{P}=0,001)$, tại chuyển đạo V2 $(80,92 \pm$ $8,91$ ms với 75,47 $\pm 3,41 \mathrm{~ms} ; P=0,022)$, chuyển đạo V3 $(80,63 \pm 5,27$ ms vs $74,18 \pm 4,8 \mathrm{~ms} ; \mathrm{P}=$ $0,000)$, tại chuyển đạo V4 $(79,46 \pm 4,10$ ms với $71 \pm 6,7 \mathrm{~ms} ; \mathrm{P}=0,000)$ và tại chuyển đạo V5 $(79,88 \pm 5,02 \mathrm{~ms}$ với $71,47 \pm 7,02 \mathrm{~ms} ; \mathrm{P}=0,000)$.

Tỉ lệ TpTe/QT cao hơn từ chuyển đạo V1 đến V5 ở nhóm bệnh nhân dương tính so với nhóm bệnh nhân âm tính trong thăm dò điện sinh lý. Tỉ lệ này tại chuyển đạo $\mathrm{V} 1(0,212 \pm 0,012$ so với $0,190 \pm 0,012 ; P: 0,000)$, tại chuyển đạo V2 $(0,213 \pm 0,012$ so với $0,191 \pm 0,011 ; P: 0,000)$, tại chuyển đạo V3 $(0,219 \pm 0,009$ so với $0,197 \pm$ 0,014; P: 0,000), tại chuyển đạo V4 $(0,224 \pm$ $0,010$ so với $0,203 \pm 0,015 ; P$ : 0,000$)$, và chuyển đạo V5 $(0,224 \pm 0,012$ so với $0,204 \pm$ 
0,02; P: 0,000). Theo nghiên cứu của Zumhagen và cộng sự thấy được rằng khoảng thời gian TpTe và tỉ lệ TpTe/QT tại chuyển đạo $\mathrm{V} 1$ dài hơn đáng kể đối với nhóm bệnh nhân có triêu chứng so với nhóm bệnh nhân không triệu chứng[7].

Trong nghiên cứu của chúng tôi, đường cong ROC đã cho điểm cut off giá trị tối ưu của khoảng thời gian TpTe và tî lệ TpTe/QT ở chuyển đạo V1 và V2 có độ nhậy và độ đặc hiệu cao hơnvới kết quả là TpTe ở V1: 76 ms cho độ nhậy $87,5 \%$ và độ đặc hiệu $76,5 \%$, TpTe ở V2 là 77,5 ms cho độ nhậy $87,5 \%$ và độ đặc hiệu $70,6 \%$. Điểm cắt của tỉ lệ TpTe/QT ở chuyển đạo V1: 0,199 cho độ nhậy 83,3\% và độ đặc hiệu 76,5\%. Ớ chuyển đạo V2 thì điểm cắt của tỉ lệ TpTe/QT là 0,201 cho độ nhậy là $87,5 \%$ và độ đặc hiệu là $88,2 \%$.

\section{KẾT LUÂ̂N}

- Khoảng thời gian Tpeak- Tend trên điện tâm đồ bề mặt dường như có một vai trò quan trọng liên quan đến rối loạn nhịp ở bệnh nhân Brugada.

- Khoảng thời gian Tpeak-Tend và tî lệ Tpeak-Tend/QT ở chuyển đạo V1 và V2 tăng cao hơn đáng kể ở những bệnh nhân Brugada gây được cơn rối loạn nhịp thất nguy hiểm trong thăm dò điện sinh lý tim.

\section{TÀI LIÊU THAM KHẢO}

1. 25 Years of Brugada Syndrome, with Dr Josep Brugada.Medscape, <http://www.medscape.com/viewarticle/895502>, accessed: 06/02/2018.

2. Brugada.J., Brugada.R., Brugada.P. (2003).Determinants of sudden cardiac death in individuals with the electrocardiographic pattern of Brugada syndrome and no previous cardiac arrest. Circulation, 108(25), 3092-3096

3. Sroubek.J., Probst.V., Mazzanti.A., et.al. (2016). Programmed ventricular stimulation for risk stratification in the Brugada syndrome. Circulation, 133(7), 622-630.

4. Antzelevitch C., Brugada P., Borgrefe M.,et al. (2005). Brugada syndrome: report of the second consensus conference: endorsed by the Heart Rhythm Society and the European Heart Rhythm Association. Circulation, (2005). 111(5), 659-670.

5. Arteyeva NV., Goshka S., Sedova K A., et al 2013. What does the $T$ (peak)- $T$ (end) interval reflect? An experimental and model study. J Electrocardiol. 46(4):291-298

6. Tokuyama.T. Nakano.Y., Awazu.A., et.al. (2014) Deterioration of the circadian variation of heart rate variability in Brugada syndrome may contribute to the pathogenesis of ventricular fibrillation. Journal of Cardiology. 64(2): 133-138.

7. Zumhagen.S., Zeidler.S.M., Stallmeyer.B. et.al. (2016). Tpeak-Tend interval and TpeakTend/QT ratio in patients with Brugada syndrome. Europace, 18(12), 1866-1872.

\title{
TÌNH TRANG THIẾU MÁU VÀ DỰ TRŨ SẮT Ở PHỤ NỮ 15-35 TUỔI TẠI MộT HUYỆN MIỀN NÚI PHÍA BẮC, NĂM 2018
}

\author{
Hoàng Nguyễn Phương Linh*, Nguyễn Hồng Trường*, \\ Nguyễn Song Tú*, Lê Danh Tuyên*
}

\section{TÓM TẮT}

Thiếu máu đang là vấn đề sức khoẻ cộng đồng tại các quốc gia có thu nhập thấp và đang phát triển. Nghiên cứu mô tả cắt ngang đã được tiến hành trên 414 phụ nữ ở độ tuổi từ $15-35$ tại 5 xã nghèo tại môt huyện miền núi phía Bắc, tỉnh Sớn La nhằm đánh giá tình trang thiếu máu, dự trữ sắt và thiếu máu thiếu sắt. Kết quả cho thấy, tỳ lệ thiếu máu là $25,6 \%$, ở mức trung binh có ý nghĩa sức khỏe cộng đồng. Tỷ lệ thiếu máu cao nhất là nhóm 15-24 tuổi (30,3\%). Tỷ lệ dự trữ sắt cạn kiệt là $15,7 \%$; dự trữ sắt thấp là $16,2 \%$; thiếu máu do thiếu sắt là $6,0 \%$ và có $19,6 \%$

${ }^{1}$ Viện Dinh dướng Quốc gia, Hà Nội

Chịu trách nhiệm chính: Hoàng Nguyễn Phương Linh Email: hoangnguyenphuonglinh@dinhduong.org.vn Ngày nhận bài: 28.7.2021

Ngày phản biện khoa học: 27.9.2021

Ngày duyệt bài: 4.10 .2021 phụ nữ thiếu máu không thiếu sắt; $9,7 \%$ thiễu sắt khổng thiếu máu. Cần tiếp tục theo dõi tình trạng thiếu máu trên phụ nữ tuổi sinh đẻ và có thễm các nghiên cứu về tìm hiểu thêm nguyên nhân dẫn đến thiếu máu để đưa ra những giải pháp can thiệp cải thiên tình trạng thiếu máu một cách chính xác và hiệu quả ở phụ nữ từ 15-35 tuổi tại các huyện miền núi phía Bắc.

Tư khóa: Thiếu máu thiếu sắt, dự trữ sắt, phụ nữ tuổi sinh đẻ, miền núi.

\section{SUMMARY}

\section{THE ANEMIA AND IRON STORES STATUS IN WOMEN AGED 15-35 YEARS OLD AT A DISTRICT IN THE NORTHERN MOUNTAINOUS AREA IN 2018}

Anemia is currently a significant public health issue in low-income, developing, and developed countries. A cross-sectional descriptive study was conducted in 414 women aged 15-35 years old in low socioeconomic in 